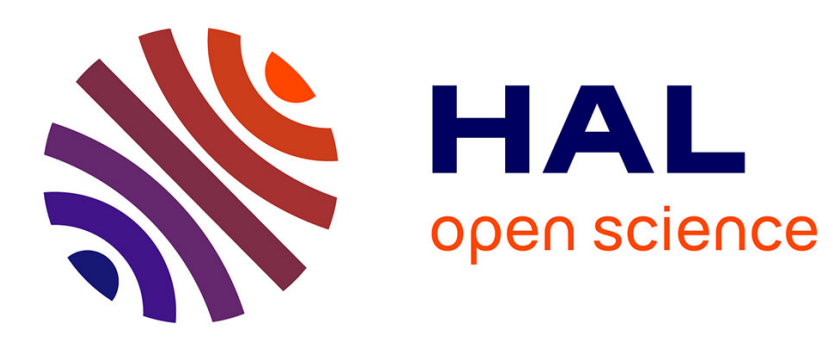

\title{
Assessment of the stability of water in water emulsions using analytical centrifugation
}

\author{
Lingsam Tea, Frédéric F. Renou, Lazhar Benyahia, Taco Nicolaï
}

\section{To cite this version:}

Lingsam Tea, Frédéric F. Renou, Lazhar Benyahia, Taco Nicolaï. Assessment of the stability of water in water emulsions using analytical centrifugation. Colloids and Surfaces A: Physicochemical and Engineering Aspects, 2021, 608, pp.125619. 10.1016/j.colsurfa.2020.125619 . hal-03026627

\section{HAL Id: hal-03026627 \\ https://hal.science/hal-03026627}

Submitted on 3 Dec 2020

HAL is a multi-disciplinary open access archive for the deposit and dissemination of scientific research documents, whether they are published or not. The documents may come from teaching and research institutions in France or abroad, or from public or private research centers.
L'archive ouverte pluridisciplinaire HAL, est destinée au dépôt et à la diffusion de documents scientifiques de niveau recherche, publiés ou non, émanant des établissements d'enseignement et de recherche français ou étrangers, des laboratoires publics ou privés. 


\title{
Assessment of the stability of water in water emulsions using analytical centrifugation
}

Lingsam Tea, Frederic Renou, Lazhar Benyahia, Taco Nicolai

Le Mans Université, IMMM UMRCNRS 6283, 72085, cedex 9, Le Mans, France

\begin{abstract}
Water in water (W/W) emulsions were prepared by mixing aqueous dextran and polyethylene oxide (PEO) solutions. Addition of different amounts of the polysaccharide chitosan inhibited coalescence of dispersed droplets of the dextran phase in the continuous PEO phasetodifferent extents depending on the concentration and $\mathrm{pH}$. Transmission profiles were measured during centrifugation and analysed. It is shown that the evolution of the transmission profile is determined by the relative rates of coalescence and sedimentation of the droplets. The rate of coalescence of the sedimented droplets into a continuous dextran phase depends on the resistance of the protective chitosan layer to compression by centrifugation. It is concluded that the efficacy of stabilization of W/W emulsions can rapidly be quantitatively assessed, but it is difficult to deduce the behaviour under gravity from that observed during centrifugation.
\end{abstract}

Keywords: water in water emulsion; aqueous two phase; analytical centrifugation; stability; turbidity

\section{Introduction}

Water in water (W/W) emulsions are obtained by mixing aqueous solutions of macromolecules that show segregative phase separation [1]. The interfacial tension between two aqueous phases is low and the interfacial thickness is larger than several nm. Therefore, W/W 
emulsions cannot be stabilized by surfactants as is commonly done for oil-water emulsions. However, they can be stabilized by particles that are large enough to span the interface and for which the interfacial tension with each phase is lower than that between the two phases [2-4]. Such particles spontaneously adsorb at the interface and can in some cases inhibit coalescence of dispersed droplets. Particle stabilized, so-called Pickering, emulsions have been studied in detail for oil/water emulsions and the driving force for the adsorption of particles at the interface has been shown to be the reduction of the interfacial tension [5].

In general, the stability of $\mathrm{W} / \mathrm{W}$ emulsions under gravity is tested by visually observing their evolution with time or by measuring the transmission profile as a function of time. Destabilization shows itself by the formation of a continuous layer of the dispersed phase. It is important here to distinguish between destabilization of the emulsions caused by coalescence and that by sedimentation or creaming of the dispersed droplets. The rate of the latter is determined by the size of the droplets and the viscosity of the continuous phase. Assessment of the stability of $\mathrm{W} / \mathrm{W}$ emulsions under gravity can be time consuming and is in practice restricted to a duration of several days. Therefore, it does not allow for discrimination between emulsions that are stable for longer durations.

It was shown that the stability of oil/water emulsions can be assessed rapidly by measuring the transmission profile over the length of the emulsion during centrifugation, see for example [611]. By analysing the profiles one can distinguish between coalescence of the droplets, on one hand, and sedimentation or creaming on the other. By varying the centrifugal force, it is also possible to assess the resistance of the particle layers around sedimented droplets to compression. As far as we are aware, the stability of $\mathrm{W} / \mathrm{W}$ emulsions has not yet been investigated using this analytical centrifugation technique. $\mathrm{W} / \mathrm{W}$ emulsions differ from oil/water emulsions by the smaller density difference between the two phases, the much lower interfacial tension and the generally larger droplet size of the dispersed phase.

Here we report on an investigation of model W/W emulsions formed by mixtures of dextran and poly(ethylene oxide) (PEO) that were more or less stabilized by the addition of different amounts of the cationic polysaccharide chitosan. Elsewhere, we have shown that addition of chitosan stabilizes the emulsions to different extents depending on the $\mathrm{pH}$, the chitosan concentration, and the composition of the mixture[12]. It was found that in the presence of $1 \mathrm{~g} / \mathrm{L}$ chitosan, the emulsions of dextran droplets in a continuous PEO phase used for the present study showed no signs of destabilization or sedimentation when standing for a week in the $\mathrm{pH}$ range 3-6, whereas in the absence of chitosan they fully phase separated macroscopically after a few hours. At 
lower chitosan concentrations, the emulsions were found to be the most stable at $\mathrm{pH} 5$. Emulsions of PEO droplets in a continuous dextran phase with the same PEO and dextran concentrations in the phases were found to be much less stable in the presence of $1 \mathrm{~g} / \mathrm{L}$ chitosan. We suggested that stabilization was caused by the formation of a layer of chitosan at the droplet surface that inhibited coalescence. However, in all cases only a small fraction of the chitosan was situated at the interface with excess chitosan dispersed in the two phases.

\section{Materials and methods}

\subsection{Materials}

PEO, dextran and chitosan were purchased from Sigma-Aldrich. Stock solutions were prepared a by dispersing in deionized water (Millipore) and stirring overnight. The PEO powder contained a small amount of silica particles that was removed by centrifuging the stock solution $(100 \mathrm{~g} / \mathrm{L})$ at $5 \times 10^{4} \mathrm{~g}$ for 4 hours and 30 minutes. The dextran stock solution was solubilized under stirring overnight at $200 \mathrm{~g} / \mathrm{L}$ and used without further purification. Chitosan was dissolved at $\mathrm{pH} 2$ $(20 \mathrm{~g} / \mathrm{L})$ in order to facilitate the solubilisation, but after a few hours stirring the $\mathrm{pH}$ was raised to 3 . Hydrolysis at pH 2 was checked by light scattering measurements and was found to be negligible at room temperature even for much longer duration. Chitosan solutions were filtered through $0.2 \mu \mathrm{m}$ Anatop filters, but filtration was found not to influence the behavior of the emulsions. The weight average molar mass was determined by light scattering as explained in ref [12]: $\mathrm{Mw}=2 \times 10^{5}, 1.6 \times$ $10^{5}$ and $3.4 \times 10^{5} \mathrm{~g} / \mathrm{mol}$ for PEO, dextran and chitosan, respectively. The acetylation degree of the chitosan sample was $25 \%$ as determined by NMR, following the method of Hirai et al. [13]. Briefly, the acetylation degree was determined by $\mathrm{NMR}$ in $\mathrm{D}_{2} \mathrm{O}$ with $\mathrm{DCl}$ at $7 \mathrm{O}^{\circ} \mathrm{C}$ to decrease the viscosity. The ratio of the integral intensity of acetyl group protons (around $2 \mathrm{ppm}$ ) and the integrated intensities of $\mathrm{H} 2$ to $\mathrm{H} 6$ (between 3,5 and 4 ppm) was used to determine the acetylation degree.

\subsection{Emulsion preparation}

Emulsions containing droplets of the dextran rich phase in the continuous PEO rich phase, were prepared by mixing $40 \mathrm{~g} / \mathrm{L}$ dextran with $63 \mathrm{~g} / \mathrm{L}$ PEO. At these conditions phase separation was almost complete with a volume fraction of $25 \%$ of the dispersed phase containing $161 \mathrm{~g} / \mathrm{L}$ dextran and $75 \%$ of the continuous phase containing $80.5 \mathrm{~g} / \mathrm{L}$ PEO in the other phase. Stock solutions of 
PEO, dextran, chitosan and water were mixed in the required amounts using a vortex stirrer. The structure of the emulsions did not depend on the order of mixing, nor on the speed or duration of vortex stirring. In fact, the same droplet size distribution was obtained by strong handshaking. The $\mathrm{pH}$ of the emulsions was adjusted by addition of small amounts of $\mathrm{HCl}$ or $\mathrm{NaOH}$ at $0.1 \mathrm{~mol} / \mathrm{L}$. The $0.1 \mathrm{M} \mathrm{HCl}$ and $\mathrm{NaOH}$ solutions were acquired from Fischer chemical. Confocal laser scanning microscopy (CLSM) images of these emulsions have already been presented in ref. [12]. We found that the number droplet diameter distribution of stable emulsions containing $1 \mathrm{~g} / \mathrm{L}$ chitosan was centred at $8 \mu \mathrm{m}$, but they contained droplets between 2 and $20 \mu \mathrm{m}$. The average diameter of nonstable emulsions without chitosan was about $20 \mu \mathrm{m}$ just after preparation and increased rapidly with time.

\subsection{Transmission profile measurements}

The transmission of light with wavelength $870 \mathrm{~nm}$ was measurement as a function of the position of the tube using commercial equipment (LUMisizer and LUMireader, LUM GmbH, Berlin, Germany). Flat bottomed rectangular polycarbonate tubes of $2 \mathrm{~mm}$ width were used to measure the transmission profiles during centrifugation with the LUMisizer and tubes of $10 \mathrm{~mm}$ width were used to measure the transmission profile under gravity with the LUMireader. The height of the liquid in the tubes was $36 \mathrm{~mm}$ for measurements with the LUMIreader and $20 \mathrm{~mm}$ with the LUMIsizer. More detailed descriptions of the apparatus can be found in refs $[6,10,11]$

\section{Results}

In first instance we will discuss the behaviour of the emulsions at $\mathrm{pH} 5$ subjected to a centrifugal force of $30 \mathrm{G}$. The effects of varying the $\mathrm{pH}$ and the centrifugal force will be discussed later. Fig. 1 shows the turbidity profiles as a function of the relative distance (d) from the bottom of the liquid in the tube at different times. Results are compared between an emulsion without stabilizer (fig. 1a) and a stabilized emulsion containing $\mathrm{C}=1 \mathrm{~g} / \mathrm{L}$ chitosan (fig. 1b). The latter emulsion did not show signs of destabilisation or sedimentation under gravity for at least one week [12]. The reproducibility of the results was tested by measuring the evolution of the profiles for independently prepared emulsions. The comparison of the profiles of different non-stabilized emulsions at different times that is shown in fig. S1 of the supplementary information is typical for all emulsions studied here. 

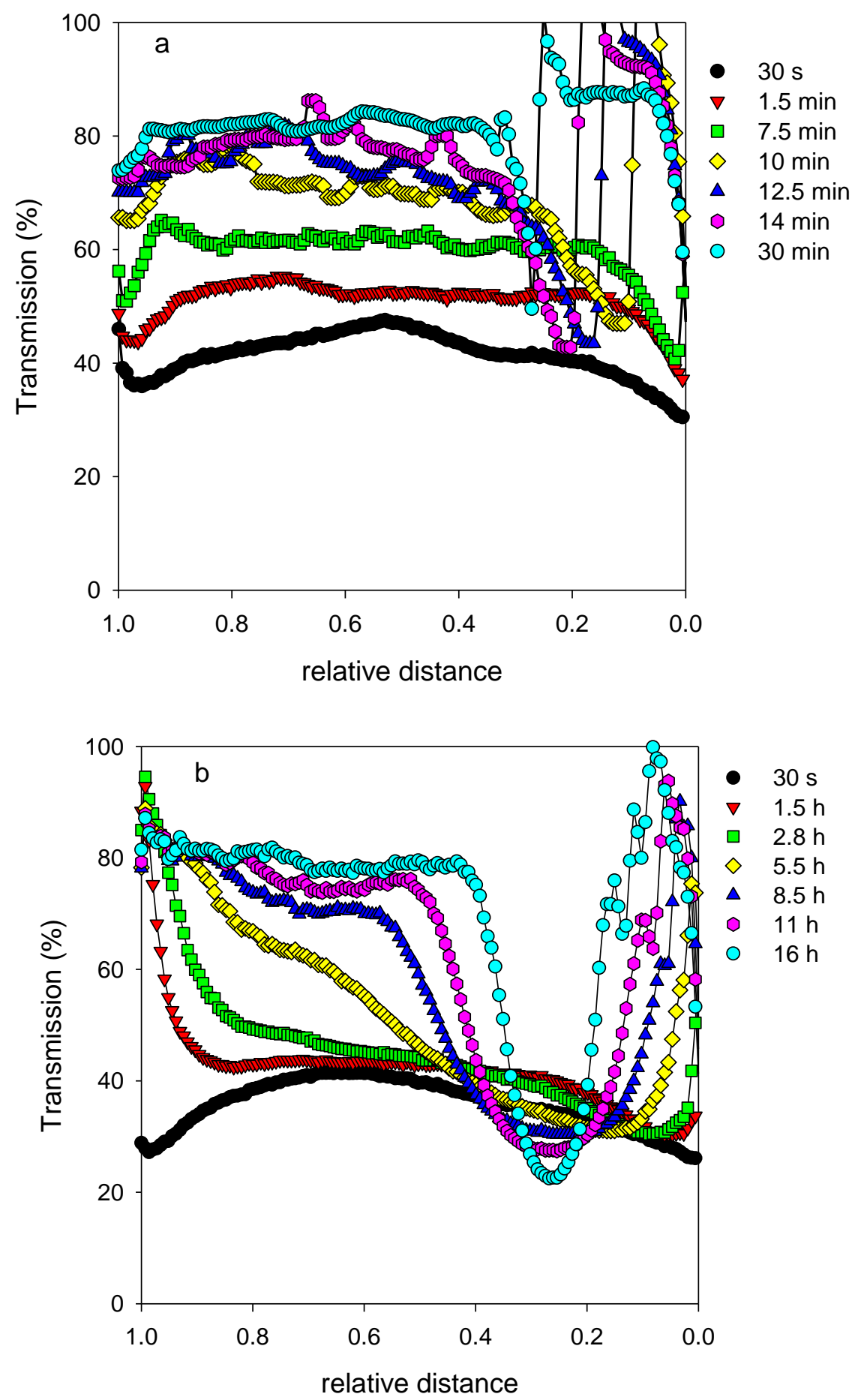

Fig. 1 Transmission profiles of emulsions without (a) and with $1 \mathrm{~g} / \mathrm{L}$ chitosan (b) at different times during centrifugation at 30G.

For the non-stabilized emulsion, one observes initially an increase of the transmission over the whole length of the liquid in the tube except near the bottom where it decreases. The decrease near the bottom is caused by accumulation of sedimenting droplets. In addition, there is an artificial 
decrease very close to the bottom caused by the way the transmitted light is detected. After $3.5 \mathrm{~min}$, an increase of the transmission is seen at the bottom of the tube that becomes rapidly more important and is caused by the formation of a clear layer of the dextran phase. The height of the dextran layer increased with time until it reached the equilibrium value $\mathrm{d}=0.25$. The transmission of the pure PEO $(\approx 81 \%)$ and dextran $(\approx 90 \%)$ layers can be gauged from the transmission profile at equilibrium.

Interestingly, the transmission of the liquid between the top and the developing bottom dextran layer increased uniformly except near the bottom layer where it sharply decreased. The latter was caused by dextran droplets accumulating on top of the bottom layer that subsequently coalesced with the continuous dextran layer increasing the height of the latter. After approximately 30 min two homogeneous phases were formed. At equilibrium one can still observe a narrow dip in the transmission between the two phases, which is caused by refraction of light at the interface.

A different evolution of the turbidity profile was found for the same emulsion in the presence of chitosan. It is important to note that addition of $1 \mathrm{~g} / \mathrm{L}$ chitosan led to an increase of the zero-shear viscosity of the emulsion from 0.4 Pa.s to 1.2 Pa.s. Therefore, if the only effect of chitosan was to increase the viscosity one might expect the same evolution as for the non-stabilized emulsions, but 3 times slower. This is clearly not what is observed. Initially, the principal change in the profile is the increase of the transmission close to the top interface due to sedimentation of the droplets. After $1.5 \mathrm{~h}$ a small increase of the transmission near the bottom was seen due to the formation of a continuous dextran phase. The growth of the bottom phase is much slower than for the nonstabilized emulsion and equilibrium was not yet reached after $16 \mathrm{~h}$, which suggests that chitosan inhibited coalescence of droplets into a continuous phase.

Contrary to the non-stabilized emulsion, there was no uniform increase of the transmission. Instead, a clear top layer consisting of the droplet free PEO phase was formed with a thickness that increased with time as the droplets sedimented. However, we did not observe the distinct front between the sedimenting droplets and the clear top phase that is observed for monodisperse colloid suspensions [14]. Instead, the transmission decreased progressively between the clear top layer and the developing continuous dextran layer at the bottom. This was most likely caused by the dispersity of the droplet size, which caused the droplets to sediment at different speeds so that a gradient of increasing droplet density was developed from the top to the bottom of the tube. The velocity of a droplet under a centrifugal force of $x . G$ is a function of the viscosity $(\eta)$ of the continuous phase, the density difference between the two phases $(\Delta \rho)$ and the radius of the droplet (R): 
$v=x . G . \Delta \rho \cdot 2 \cdot R^{2} /(9 . \eta)$. Using the density difference between the phases $\Delta \rho=50 \mathrm{~kg} / \mathrm{m}^{3}$ reported in ref. [15] and $\eta=1.2$ Pa.s, we find $v=1.5 \times 10^{-6} . x \mathrm{~mm} / \mathrm{s}$ for the initial average droplet radius $(4 \mu \mathrm{m})$. This would mean that at $30 \mathrm{G}$ the droplets sediment over half the length of the liquid ( $10 \mathrm{~mm}$ ) after $2 \times 10^{5}$ $\mathrm{s}$, which is about 6 times longer than the experimentally observed time, reflecting the effect of interactions and droplet growth.

With time, the top layer became depleted of droplets and a dense layer of droplets was formed on top of the continuous dextran layer causing the distinct minimum in the transmission. This turbid intermediate layer decreased in size from the bottom as the droplets coalesced with the continuous dextran layer and from the top as the droplets in the layer became more compacted. Visually one can clearly observe a turbid layer between two clear layers that slowly decreases in thickness, see fig. S2 of the supplementary information. The top layer took a long time to completely clarify because it contained small droplets that took a long time to sediment.

It is clear that the behaviour of the non-stabilized W/W emulsion cannot be understood by sedimentation of the droplets followed by coalescence of the compressed droplets. It is not straightforward to understand the almost uniform increase of the transmission of the top layer. The principal difference between stabilized and non-stabilized emulsions is that non-stabilized droplets immediately coalesce when they collide as was shown elsewhere by confocal laser scanning microscopy (CLSM) [12]. Coalescence leads to larger droplets and, as a consequence an increase of the amount of scattered light. However, larger droplets scatter light at smaller angles with the transmitted light. The transmitted light is, of course, always detected over a finite angular range in the forward direction. We don't know the detected angular range of the equipment used here, but if it is few degrees a large fraction of the light scattered by droplets larger than $10 \mu \mathrm{m}$ would be detected as transmitted light. This explains why the transmission increases when droplets coalesce. However, it does not explain why the transmission increases uniformly over the whole distance. Fig. $1 \mathrm{~b}$ shows that the sedimentation rate of stabilized droplets that remain small is negligible on the time scale where non-stabilized emulsions phase separate. This means that in non-stabilized emulsions only larger droplets sedimented. We speculate that in non-stabilized emulsions smaller droplets increased in size by coalescence until their sedimentation rate was no longer negligible on the time scales of the experiment. Once droplets start to sediment the probability to encounter other, smaller, droplets increases, which accelerates their growth. Since, as we mentioned above, the effect of these large sedimenting droplets on the transmission is relatively small compared to that of the smaller very slowly sedimenting droplets, it will not be apparent on the transmission profile. 
The two processes of sedimentation and coalescence of the dextran droplets within the PEO phase occur concomitantly, which is illustrated in fig. 2 where we show the evolution of the turbidity profiles for an emulsion containing $0.6 \mathrm{~g} / \mathrm{L}$ chitosan, with intermediate stability. Initially the transmission increased uniformly as for the non-stabilized emulsions, but after $17 \mathrm{~min}$ a sedimentation front was formed that sharpened with time and moved to the bottom of the tube. For this emulsion equilibrium was reached after 16h. Included in fig. 2 are cartoons that illustrate the morphology of the emulsion at different times during centrifugation.

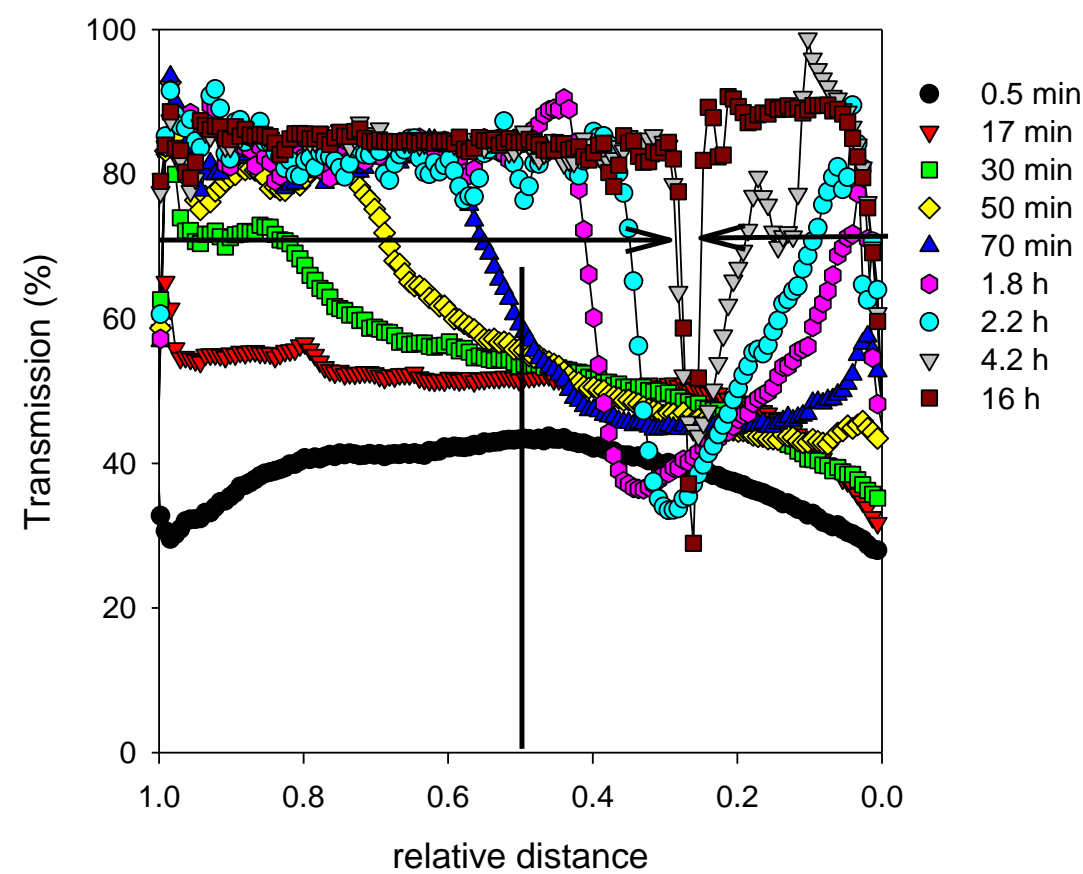

relative distance

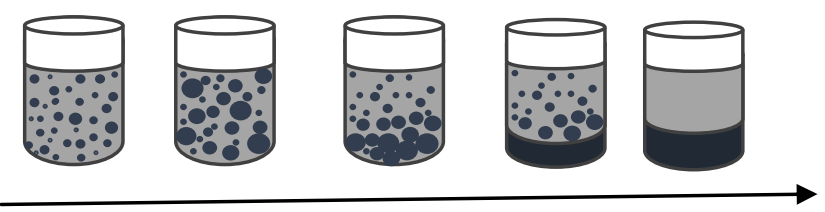

time

Fig. 2 Transmission profiles of emulsions with $0.6 \mathrm{~g} / \mathrm{L}$ chitosan at different times during centrifugation at $30 \mathrm{G}$. The horizontal arrows illustrate the shift with time of the fronts of the clear PEO and dextran layers at a transmission of $70 \%$. The vertical arrows illustrate the increase of the transmission with time at $d=0.5$. Cartoons that illustrate different stages of the emulsion during centrifugation are also shown. 


\subsection{Quantitative analysis}

In order to quantify the evolution of the layer formation, we determined the time when the transmission reached a certain value as a function of the position in the tube as illustrated by the horizontal arrows in fig.2. This is particularly useful when the border between layers is well-defined and indicated by a sharp decrease of the transmission. Its meaning is more ambiguous when the interface is not sharp and when coalescence of droplets occurs within the continuous phase. Nevertheless, the speed at which the layers are formed can still be quantified in this manner. Fig. 3 shows $d$ as a function of time for the fronts of the top and bottom layer measured at $70 \%$ transmission for emulsions containing different chitosan concentrations. Notice that because the front is not sharp, the layers already have a certain thickness when the given transmission is reached. With time, the top and bottom fronts move to smaller and larger $d$, respectively, until they meet at the equilibrium value of $\mathrm{d} \approx 0.25$. The lower the chosen transmission the sooner this transmission is reached at a given position and the sooner the equilibrium value of the position is reached, see fig. S3 of the supplementary information. However, the relative variation of the front position is approximately independent of the chosen transmission value and is characteristic for the speed at which the layers are formed. Most reliable results were obtained if the transmission was chosen close, but not too close, to the transmission of the phases at equilibrium.

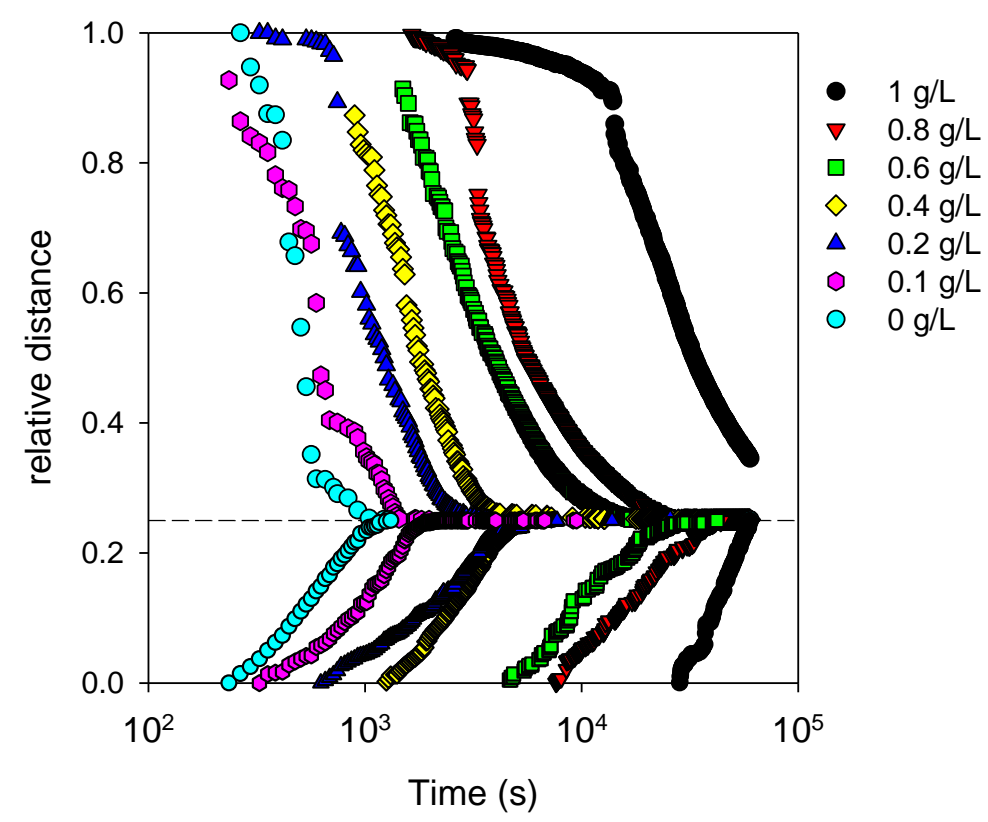

Fig. 3. Relative positions in the tube of the fronts of the clear PEO and dextran layers for emulsions with different chitosan concentrations as a function of centrifugation time at $30 \mathrm{G}$ measured at $70 \%$ transmission. 
The rate at which droplets coalesce in the continuous PEO phase can be quantified by measuring the transmission as a function of time as illustrated by the vertical arrow in fig.2. Of course, this is useful only as long as the transmission increases uniformly and a sedimentation front is not yet formed. Fig. 4 shows the average transmission between $d=0.43$ and 0.67 . Initially, the transmission increased logarithmically with a rate that decreased with increasing chitosan concentration. This progressive increase was interrupted by a sharp increase that was caused by the fact that the sedimentation front traversed the chosen range of $d$. The rate at which the transmission increases before the sharp rise is characteristic for the rate of coalescence and the time at which it increases sharply is characteristic for the rate of sedimentation. The two analysis methods discussed here allow for a quantitative description of the rate of droplet sedimentation, formation of the continuous layer of the dispersed phase and coalescence the dispersed droplets.

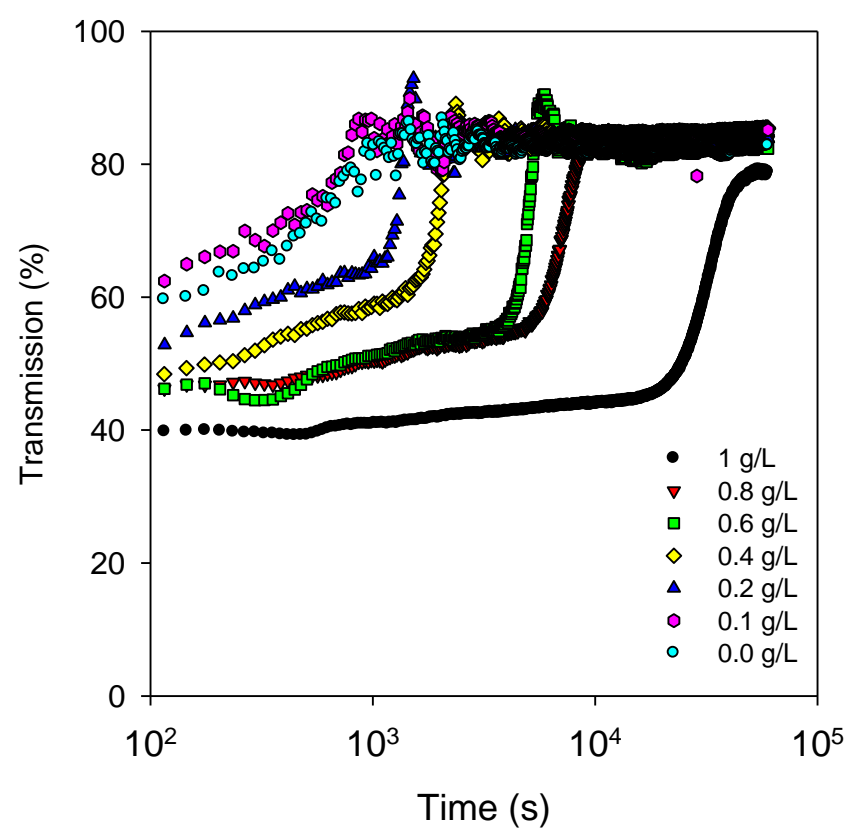

Fig. 4 Evolution of the average transmission between $d=0.43$ and $d=0.67$ of DP emulsions at different chitosan concentrations during centrifugation at 30G.

\subsection{Effect of the chitosan concentration}

It is clear from fig.3 that the PEO and dextran layers developed more slowly when more chitosan was present. A characteristic time $\left(t_{c}\right)$ was defined as the time when the thickness of the evolving top and bottom layers reached half that of the layers at equilibrium, i.e. at $\mathrm{d}=0.725$ and 0.125, for the PEO and dextran layer, respectively. Fig. 5 shows the increase of $t_{c}$ with increasing concentration of chitosan for the top and the bottom layer. Values of $t_{c}$ obtained from independent 
measurements at each chitosan concentration are plotted, which allows one to estimate the experimental uncertainty. The characteristic time increased sharply with increasing chitosan concentration, but there does not appear to be a critical concentration that separates unstable from stable emulsions. The time characterizing the formation of the continuous dextran layer was longer than that characterizing the formation of the clear PEO layer. This means that sedimentation of the droplets was faster than their coalescence into a continuous dextran phase. Notice, however, that at low chitosan concentrations ( $\mathrm{C}<0.2 \mathrm{~g} / \mathrm{L}$ ) coalescence of dextran droplets within the PEO phase was relatively fast compared to sedimentation and no clear sedimentation front was developed. For comparison the relative increase of the viscosity with increasing chitosan concentration is also shown in fig. 5 . It was much weaker and therefore cannot explain the increase of $t_{c}$.

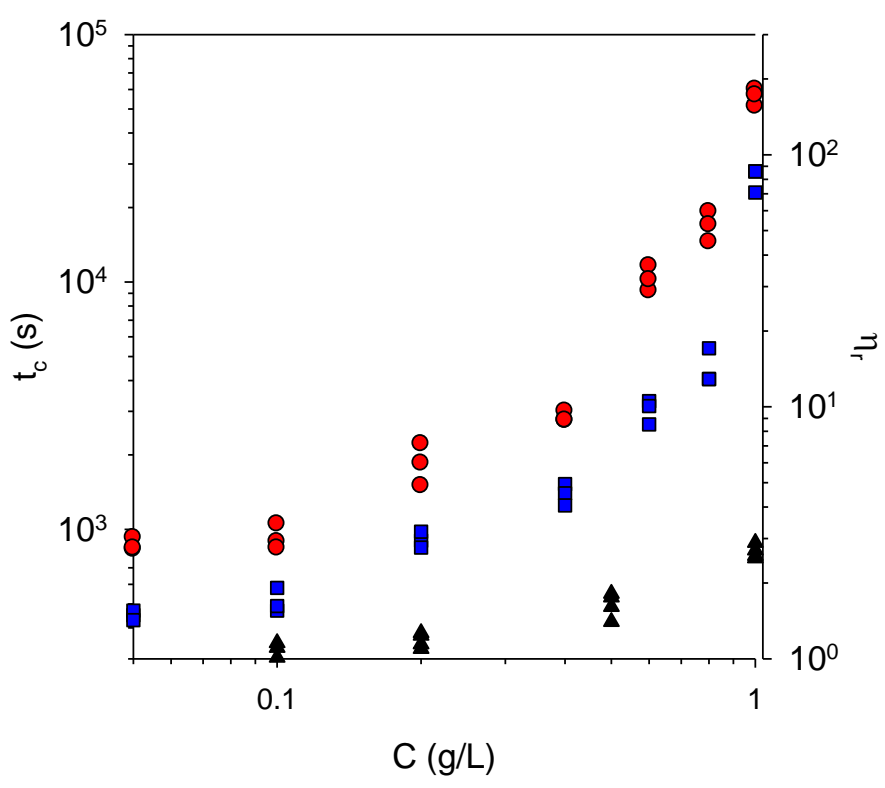

Fig. 5 Characteristic times for the formation of the clear PEO (squares) and dextran (circles) phases in emulsions during centrifugation at $30 \mathrm{G}$ as a function of the chitosan concentration. The relative increase of the viscosity is also shown (triangles) on the same relative scale in order to facilitate comparison.

The effect of the chitosan concentration on the rate of coalescence of droplets dispersed in the PEO phase is shown in fig. 4. The rate at which the transmission increased slowed down strongly with increasing chitosan concentration and became almost imperceptible at $\mathrm{C}=1 \mathrm{~g} / \mathrm{L}$. The initial slow increase was followed by a sharp increase indicating the moment that the sedimentation front passed, which occurred later when more chitosan was present. The time when the average transmission rises rapidly characterizes the sedimentation rate and has the same dependence on the chitosan concentration as $t_{c}$ plotted in fig. 5 Notice that the sharp increase is not clearly observed for 
non-stabilized emulsions and at $\mathrm{C}=0.1 \mathrm{~g} / \mathrm{L}$, because the effect of sedimentation was small compared to that of coalescence, see fig. 1a. The sharp increase started at lower transmission with increasing $\mathrm{C}$, because coalescence was slower and the droplets remained smaller. The presence of smaller droplets is the principle reason why sedimentation slows down by orders of magnitude when more chitosan is present, the effect of increasing viscosity being only a minor factor.

\subsection{Effect of the $p H$}

Chitosan has a pKa of 6.5 and its charge density decreases with increasing $\mathrm{pH}$. Above $\mathrm{pH} 6$ the charge density is no longer sufficient to avoid aggregation of chitosan chains and for $\mathrm{pH}>6.8$ macroscopic precipitation of the chitosan was observed. The effect of the $\mathrm{pH}$ on the evolution of the emulsions was studied at $\mathrm{pH} 3,4,5$ and 6 . We note that the droplet size distribution was little influenced by the $\mathrm{pH}$. Qualitatively the same phenomena were observed at all $\mathrm{pH}$. At $\mathrm{pH} 4,5$ and 6, the rate of sedimentation and formation of the continuous dextran layer was the same within the experimental spread, see fig. S4 of the supplementary information. However, at $\mathrm{pH} 3$ the emulsions evolved significantly faster in particular at low chitosan concentrations. It is difficult to explain the effect at $\mathrm{pH} 3$ by differences in charge density of chitosan, which is small between $\mathrm{pH} 3$ and $\mathrm{pH} 4$, whereas it is much bigger between $\mathrm{pH}_{4}$ and $\mathrm{pH} 6$. In addition, the viscosity was not much influenced by the $\mathrm{pH}$. We speculate that the effect was caused by a change in the interaction between chitosan and the PEO and/or dextran phase. Indeed, we found that the preferential partitioning of excess chitosan to the dextran phase decreased when the $\mathrm{pH}$ was decreased from 6 to 3 [12], which indicates a change in the interaction. However, the change was progressive and not particularly large between $\mathrm{pH} 4$ and $\mathrm{pH} 3$.

\subsection{Effect of the centrifugal force}

So far only results at a single centrifugal force have been shown. An important issue is the influence of the centrifugal force on the evolution of the emulsions. For dilute colloid suspensions, the relationship between the sedimentation rate and the force is straightforward as long as movement by Brownian diffusion is negligible compared to movement induced by the centrifugal force. For non-dilute suspensions it is less straightforward, because hydrodynamic interactions between the particles will depend on their velocity. For $\mathrm{W} / \mathrm{W}$ emulsions matters are further complicated by the fact that the droplets can coalesce and therefore change in size. The probability of two colliding droplets to coalesce is not expected to depend much on the centrifugal force until 
310 the droplets are densely packed at the bottom. Therefore, one expects that the difference between 311 rates of coalescence and sedimentation depends on the centrifugal force.

The effect of centrifugal force is illustrated in fig. 6 where the evolution of the transmission profiles is compared for non-stabilized emulsions during centrifugation at $1 \mathrm{G}$ and $10^{3} \mathrm{G}$. We note that for practical reasons the measurements at $1 \mathrm{G}$ were done in different tubes, which explains why the initial transmission was lower. At $1 \mathrm{G}$, the transmission increased uniformly implying that coalescence was fast compared to sedimentation. Visually, one observes this as clarification of the suspension even before the appearance of a clear bottom phase and the absence of a clear top phase distinct from a turbid layer of sedimenting droplets. At $10^{3} \mathrm{G}$, a sedimentation front develops within seconds before the dispersed droplets coalesce to a significant extent. Comparison with fig. 1 shows that the difference at different centrifugal forces for a given emulsion is similar to the difference between different chitosan concentrations at the same force. It is in both cases caused by the change in the ratio of the sedimentation and coalescence rates. For non-stabilized emulsions the rate of formation of the dextran layer is directly coupled to the rate of sedimentation, because the droplets immediately coalesce into a continuous phase when they reach the bottom. 

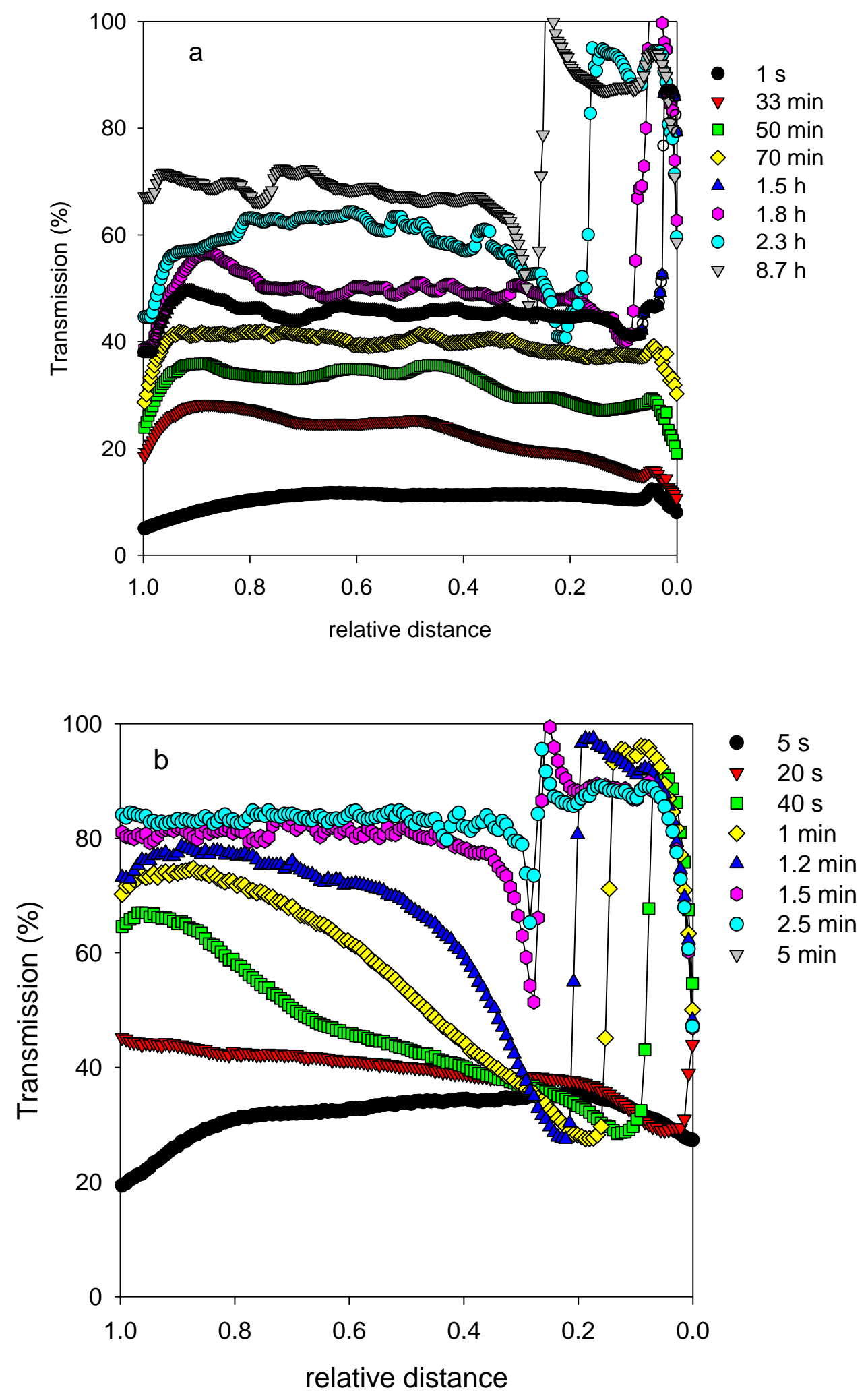

Fig. 6 Transmission profiles of non-stabilized emulsions at different times during centrifugation at $1 \mathrm{G}(\mathrm{a})$ and $10^{3} \mathrm{G}(\mathrm{b})$. 
In the presence of $1 \mathrm{~g} / \mathrm{L}$ chitosan, coalescence of dextran droplets dispersed in the PEO phase was extremely slow and sedimentation at $1 \mathrm{G}$ was also extremely slow, because the droplets remained small. As a consequence, hardly any change in the transmission was observed during a period of at least 12 days. At $10^{3} \mathrm{G}$, sedimentation did occur, but it was much slower than for the non-stabilized emulsion at this centrifugal force, because the droplets remained small, see fig. 7. In this case one can clearly see the formation of a dense layer of dextran droplets at the bottom in contact with a continuous dextran phase formed by destabilization of the droplets that evolved very slowly. It shows that droplets surrounded by a chitosan layer can resist to some extent to coalescence with each other and with the continuous dextran phase even if they are compacted by a centrifugal force.

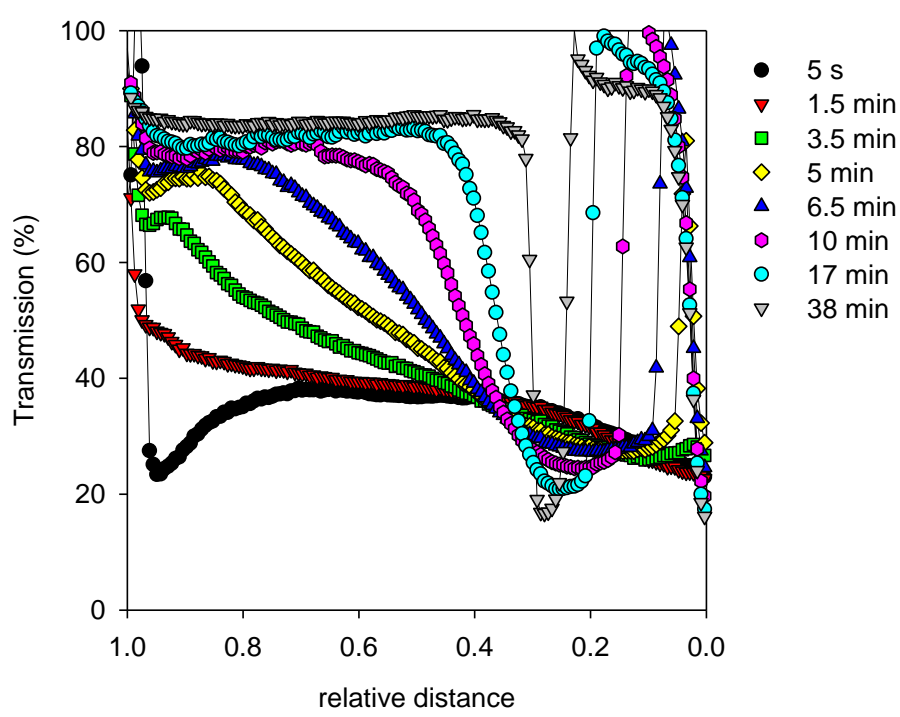

Fig. 7 Transmission profiles of emulsions at $\mathrm{C}=1 \mathrm{~g} / \mathrm{L}$ at different times during centrifugation at $10^{3} \mathrm{G}$.

The effect of the centrifugal force on the evolution of the emulsions was compared quantitatively by measuring $t_{c}$ as a function of the chitosan concentration at 30,150 and $10^{3} \mathrm{G}$, see fig. 8. As expected, stronger centrifugal force led to faster sedimentation. However, the results did not superpose when $t_{c}$ was multiplied with the force as would be the case for dilute monodisperse colloid suspensions, demonstrating the effects of coalescence and hydrodynamic interactions. The continuous dextran phase formed much faster at $10^{3} \mathrm{G}$, but interestingly the rate was almost the same at 30 and $150 \mathrm{G}$. This suggests that at $10^{3} \mathrm{G}$ the centrifugal force contributed to the coalescence of sedimented droplets with the continuous dextran phase at the bottom. It would explain why at $10^{3} \mathrm{G}$ the development of the continuous dextran phase was not much slower than sedimentation. 



It is clear from this comparison that the centrifugal force influences the evolution of the emulsions and that one cannot derive quantitatively the behaviour under gravity from that during centrifugation. The main reason for this is that coalescence and sedimentation depend differently on the centrifugal force. As we discussed in detail in ref. [12], we believe that the chitosan adsorbs at the interface driven by a reduction of the interfacial tension similar to the mechanism proposed for Pickering emulsions that was mentioned in the introduction even though we found that the reduction was very small [15]. However, in all cases only a small fraction of the added chitosan is adsorbed at the interface and the majority is partitioned between the two phases. This means that the droplet size as a function of the chitosan concentration is not controlled by limited coalescence. More likely, it takes time to form a layer that is sufficiently dense and structured to inhibit coalescence even at high chitosan concentrations. This means that initially the droplets can coalesce, but that the coalescence rate decreases with time. This will also lead to a different evolution of the emulsion at different centrifugal forces. Fast coalescence of droplets in the presence of chitosan after cessation of shear was observed by CLSM [12]. It explains why at C $=1 \mathrm{~g} / \mathrm{L}$ the dextran droplets are stable, but not very small even though there is a large excess of chitosan. It also explains why even at large chitosan concentrations we still observe initially a fast small increase of the transmission that arrests and is followed by sedimentation. The shear rate dependent viscosity of these emulsions as well as the microstructure under shear flow was reported in ref. [15]. It was found that under shear flow thin elongated strands were formed that rapidly broke up into very small droplets after 
cessation of the shear. These droplets initially quickly grew in size by coalescence, but the rate of coalescence decreased in the presence of chitosan and a steady state size was reached within a few minutes.

We may compare the results obtained here for $\mathrm{W} / \mathrm{W}$ emulsions with reported investigations of the stability of $\mathrm{O} / \mathrm{W}$ emulsions using the same method. Bardolato et al. [6] observed that the stability of $\mathrm{O} / \mathrm{W}$ emulsions containing different surfactants that were stable for one month at $1 \mathrm{G}$ could be distinguished by the different creaming rate at $1100 \mathrm{G}$. They concluded that creaming rates differed because the surfactants were more or less effective to inhibit coalescence. Sobich and Lerche [10] found for an $\mathrm{O} / \mathrm{W}$ emulsion containing surfactant that initially the oil droplets creamed leading to a turbid top and transparent bottom layer. However, with time the transmission at the top increased as the droplets coalesced and formed a continuous oil layer. They found that the creaming rate was proportional to the centrifugal force for emulsions for which coalescence was negligible during sedimentation. Analytical centrifugation has often been used to determine the creaming stability of $\mathrm{O} / \mathrm{W}$ emulsions that do not show significant coalescence. The creaming velocity is measured as a function of the centrifugal force and extrapolated to 1 G. However, extrapolation can be difficult, because the velocity is not always found to be proportional $[7,8]$. The striking interplay between sedimentation/creaming of droplets and their coalescence during this process that was observed here for $\mathrm{W} / \mathrm{W}$ emulsions has, as far as we are aware, not been noted for $\mathrm{O} / \mathrm{W}$ emulsions. Probably, because the method was used mainly to characterize the long time sedimentation/creaming stability of emulsions that did not show coalescence.

\section{Conclusion}

Here we showed for the first time that the stability of W/W emulsions can be quantitatively characterized using analytical centrifugation. Three phenomena can be distinguished: coalescence of dispersed droplets, sedimentation (or creaming) of dispersed droplets and coalescence of sedimented (creamed) droplets into a continuous phase. The relative rate of these phenomena determines the evolution of the transmission profiles and depends on the applied centrifugal force. No sedimentation front is observed when coalescence is rapid compared to sedimentation of the small droplets in the emulsion, whereas larger centrifugal forces may force sedimentation before coalescence becomes prevalent. Therefore, it is not possible to deduce the evolution of the emulsions under gravity from results obtained at larger centrifugal forces. However, it is possible to rapidly draw conclusions about the efficacy of the addition of particles or polymers on the stability of the 
emulsions. One can also gauge the resistance of the particle layer around the droplet to compression.

Coalescence of dextran droplets dispersed in a PEO solution is increasingly inhibited when more chitosan is added by the formation of stabilizing layer of the latter around the droplets. Sedimentation is also slower when more chitosan is present, because the droplets grow more slowly.

\section{References}

[1] W.J. Frith, Mixed biopolymer aqueous solutions - phase behaviour and rheology, Adv. Colloid Interface Sci., 161 (2010) 48-60.

[2] E. Dickinson, Particle-based stabilisation of water-in-water emulsions containing mixed biopolymers, Trends in Food Science \& Technology, (2018).

[3] J. Esquena, Water-in-water (W/W) emulsions, Current Opinion in Colloid \& Interface Science, 25 (2016) 109-119.

[4] T. Nicolai, B. Murray, Particle stabilized water in water emulsions, Food Hydrocolloids, 68 (2016) 157-163.

[5] R. Aveyard, B.P. Binks, J.H. Clint, Emulsions stabilized solely by colloidal particles, Adv. Colloid Interface Sci., 100 (2003) 503-546.

[6] G. Badolato, F. Aguilar, H. Schuchmann, T. Sobisch, D. Lerche, Evaluation of long term stability of model emulsions by multisample analytical centrifugation, Surface and interfacial forces-from fundamentals to applications, Springer2008, pp. 66-73.

[7] I. Dammak, P.J. do Amaral Sobral, Formulation and stability characterization of rutin-loaded oil-in-water emulsions, Food Bioprocess Technology, 10 (2017) 926-939.

[8] A.J.C. Tehrani-Bagha, S.A. Physicochemical, E. Aspects, Cationic gemini surfactant with cleavable spacer: Emulsion stability, 508 (2016) 79-84.

[9] D. Xu, X. Wang, J. Jiang, F. Yuan, Y.J.F.H. Gao, Impact of whey protein-Beet pectin conjugation on the physicochemical stability of $\beta$-carotene emulsions, 28 (2012) 258-266.

[10] T. Sobisch, D. Lerche, Rapid characterization of emulsions for emulsifier selection, quality control and evaluation of stability using multisample analytical centrifugation, $\mathrm{SCl} / \mathrm{RSC} / \mathrm{SCS}$ conference on cosmetics and colloids, London, 15 Feb 2005. http://www.soci.org/SCl/groups/col/2005/reports/pdf/gs3257 sob.pdf

[11] S. Küchler, C. Schneider, D. Lerche, T.J.L.I. Sobisch, Process optimisation for making stable emulsions using accelerated dispersion analysis by multisample analytical centrifugation, 20 (2006).

[12] L. Tea, T. Nicolai, F.J.L. Renou, Stabilization of Water-In-Water Emulsions by Linear Homo-Polyelectrolytes, 35 (2019) 9029-9036.

[13] A. Hirai, H. Odani, A. Nakajima, Determination of degree of deacetylation of chitosan by 1 H NMR spectroscopy, Polymer Bulletin, 26 (1991) 87-94.

[14] D. Lerche, Dispersion Stability and Particle Characterization by Sedimentation Kinetics in a Centrifugal Field, Journal of Dispersion Science and Technology, 23 (2002) 699-709.

[15] L. Tea, T. Nicolai, L. Benyahia, F. Renou, Viscosity and Morphology of Water-in-Water Emulsions: The Effect of Different Biopolymer Stabilizers, Macromolecules, (2020). 